\title{
A CASE OF HYPERSENSITIVITY PNEUMONITIS IN A WORKER EXPOSED TO TEREPHTHALIC ACID IN THE PRODUCTION OF POLYETHYLENE TEREPHTHALATE
}

\section{PIETRO SARTORELLI ${ }^{1}$, GABRIELE D'HAUW ${ }^{1}$, DONATELLA SPINA ${ }^{2}$, LUCA VOLTERRANI ${ }^{3}$, and MARIA ANTONIETTA MAZZEI ${ }^{3}$}

${ }^{1}$ University of Siena, Siena, Italy

Department of Medical Biotechnology, Unit of Occupational Medicine, University Hospital of Siena

${ }^{2}$ University Hospital of Siena, Siena, Italy

Unit of Pathology

${ }^{3}$ University of Siena, Siena, Italy

Department of Medicine, Surgery and Neuroscience, Unit of Diagnostic Imaging, University Hospital of Siena

\begin{abstract}
Occupational hypersensitivity pneumonitis (OHP) is an interstitial lung disease caused by sensitization to an inhaled antigen. Polyethylene terephthalate (PET) is mainly used for disposable beverage bottles. A clinical case of hypersensitivity pneumonitis (HP) in a 66-year-old patient in the follow-up as a worker formerly exposed to asbestos is presented. At the first visit in 2012 a diagnosis of asbestosis and pleural plaques was formulated. In 2017 the high resolution computed tomography was performed demonstrating a slight progression of the pulmonary fibrosis, while physical examinations revealed inspiratory crackles on auscultation, and lung function tests showed a decreased diffusing capacity for carbon monoxide. The radiological and histological pictures were compatible with HP. From 1992 to 2013 the patient worked in a chemical company that produced PET for disposable beverage bottles. A diagnosis of OHP was made, and the most likely causative agents were terephthalic acid and dimethyl terephthalate. To the best of the authors' knowledge, this is the first report of an OHP case in PET production. Int J Occup Med Environ Health. 2020;33(1):119-23
\end{abstract}

Key words:

asbestos, extrinsic allergic alveolitis, polyethylene terephthalate, terephthalic acid, pulmonary fibrosis, pleural plaques

\section{INTRODUCTION}

According to the position paper of the European Academy of Allergy and Clinical Immunology (EAACI) [1], occupational hypersensitivity pneumonitis (OHP) "is an immunologic lung disease with variable clinical presentation and outcome resulting from lymphocytic and frequently granu- lomatous inflammation of the peripheral airways, alveoli, and surrounding interstitial tissue which develops as the result of a non-IgE-mediated allergic reaction to a variety of organic or low molecular weight agents that are present in the work environment." As demonstrated for idiopathic pulmonary fibrosis (IPF), a multidisciplinary approach in-

Funding: publication of this work was supported by the Tuscan Region (project DGRT 396/2016, entitled "Health surveillance for former asbestos exposed workers," project manager: Elisabetta Chellini, Ph.D.).

Received: April 16, 2019. Accepted: September 10, 2019.

Corresponding author: Pietro Sartorelli, University of Siena, Department of Medical Biotechnology, Unit of Occupational Medicine, University Hospital of Siena, Viale Bracci 16, 53100 Siena, Italy (e-mail: pietro.sartorelli@unisi.it). 
cluding clinicians, radiologists, pathologists, and occupational physicians is strongly recommended to improve the diagnosis of OHP [2]. Today the frequency of some well known forms of the disease, such as the farmer's lung, has decreased, while new causal agents are observed.

Polyethylene terephthalate (PET) is a clear, strong and lightweight thermoplastic polymer resin of the polyester family, mainly used for packing liquids and foods. It is produced from ethylene glycol (EG) and terephthalic acid (TPA) or dimethyl terephthalate (DMT). The first step in the polymerization sequence is an esterification of acid with glycol, with water being liberated in the process. The second step in the polymerization sequence is the polycondensation of bis-hydroxyethyl terephthalate (BHET) with the liberation of EG. Many catalysts are used during the polycondensation as well as during the initial esterification or transesterification (e.g., antimony compounds such as trioxide or triacetate). Certain health effects have been recognized for phthalates and derivatives. In particular, a number of reports have suggested that endocrine disruptors may leach into the contents of bottles made from PET [3].

A clinical case of a patient with a history of both occupational asbestos and TPA exposure is presented. During the follow up as an asbestos-exposed worker, the diagnosis of OHP was formulated.

\section{CASE REPORT}

The patient was a 66-year-old male, non-smoker, in the follow-up since 2012 as a worker formerly exposed to asbestos. He worked as a maintenance mechanic in the ship engine room in 1973-1992. His work included the removal of asbestos insulation of various parts of the engine room before repairing the installations and insulating them again with asbestos. In 1992-2013 he worked in a chemical company that produced PET for disposable beverage bottles. He worked at the extrusion line of PET sheets where he was exposed to fumes produced in the polymerization process.
At the first visit in 2012, the patient's ventilatory capacity was not reduced, even if he reported symptoms of chronic bronchitis (i.e., cough and phlegm for at least 3 months/year) in the last 15 years. The clinical examination of the chest was negative. The high resolution computed tomography (HRCT) showed pulmonary fibrosis with subtle thickening of the inter- and intra-lobular septa in the subpleural regions of both lungs. Pleural thickening was also present (Figure 1a). The mineralogical analysis of the bronchoalveolar lavage fluid (BALF) by scanning electron microscopy (SEM) showed an amphiboles concentration of $472 \mathrm{ff} / \mathrm{ml}$ BALF, while chrysotile fibres and asbestos bodies were under the detection limit. This result was comparable with the concentration levels of amphiboles in BALF, measured in the same laboratory in patients suffering from asbestosis [4]. At the end of the investigations, a diagnosis of asbestosis and pleural plaques (PPs) was formulated.

In 2017, during the follow-up for asbestosis and PPs related to the past asbestos exposure, another HRCT examination was performed demonstrating a slight progression of the pulmonary fibrosis not only in the extension but also in the profusion of CT features, both in the upper and lower lobes, in relation to the previous HRCT performed in 2015 (Figure 1b). Physical examinations revealed inspiratory crackles at the pulmonary bases on auscultation. The patient recently experienced the onset of progressive dyspnea. Moreover, his lung function tests showed a decreased diffusing capacity for carbon monoxide (CO). The mineralogical analysis showed an amphiboles concentration of $320 \mathrm{ff} / \mathrm{ml}$ BALF (chrysotile fibres and $\mathrm{AB}$ were again under the detection limit). The total number of cells in BALF was $90700 / \mathrm{ml}$, with $71 \%$ of macrophages, $14 \%$ of lymphocytes, $6 \%$ of neutrophils and $9 \%$ of eosinophils, and with a $\mathrm{CD} 4 / \mathrm{CD} 8$ ratio of 1.68 .

For a better definition of the case, pneumological advice was requested that suggested performing a surgical lung biopsy. The histological examination carried out on the lung biopsies showed bronchiolocentric lymphocytic interstitial pneumonitis with scattered eosinophils and an orga- 
a)

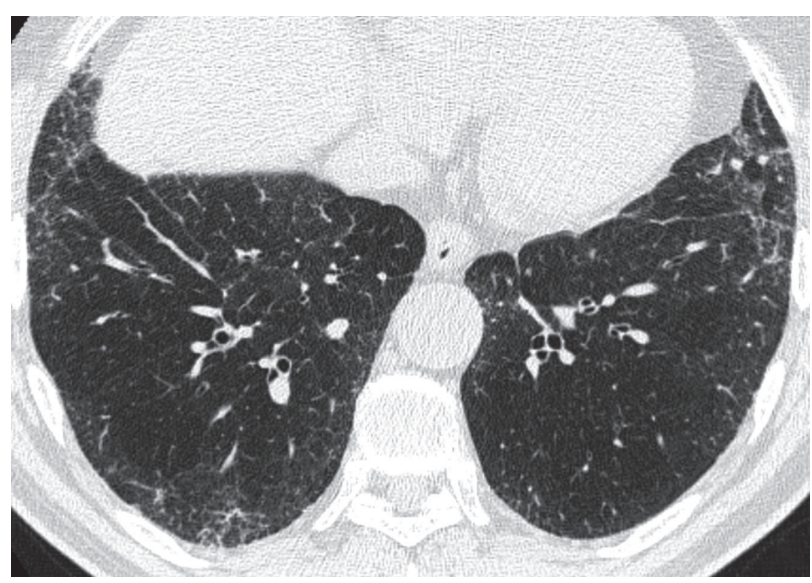

b)

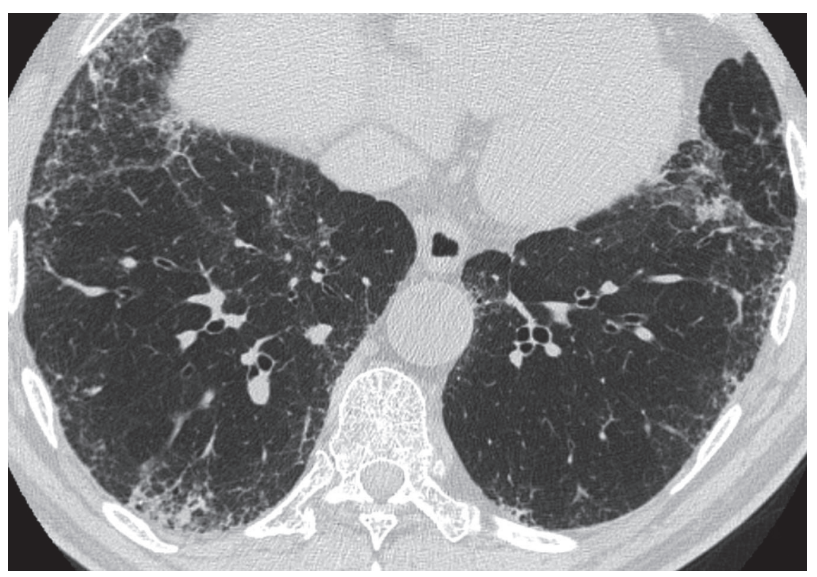

c)

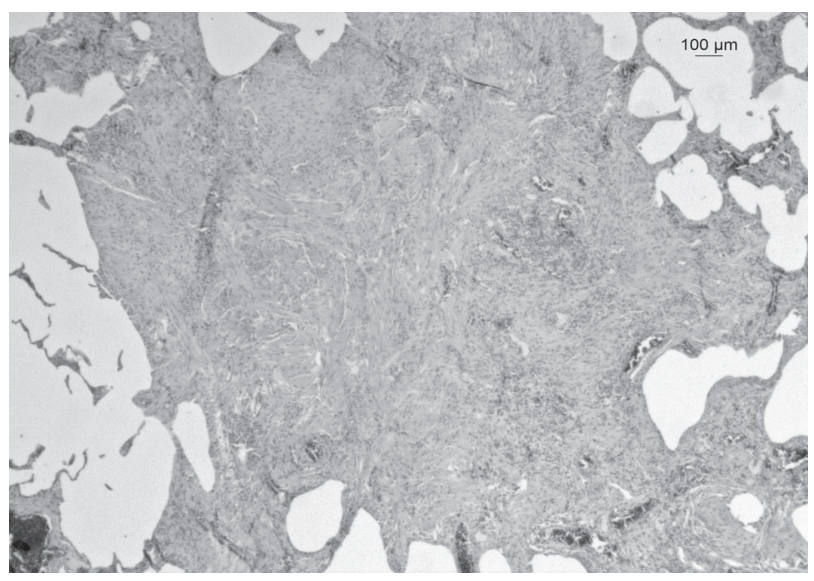

d)

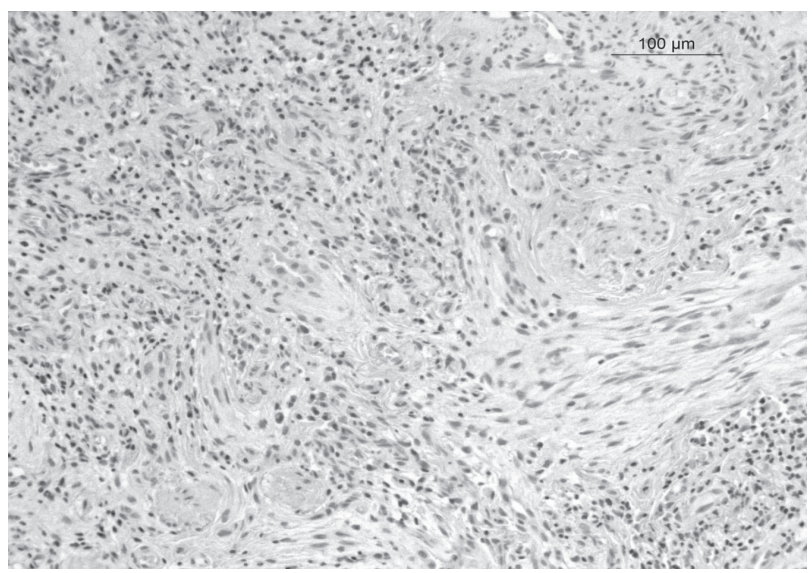

Figure 1. Examinations of a 66-year-old patient, a worker formerly exposed to asbestos. High resolution computed tomography scans showing a moderate increase in fibrotic changes in 2 different CT, performed in a) 2012 (the early fibrotic changes were distributed in the subpleural region, along the peribronchiolar structures) and b) 2015. Histological specimens confirmed c) a centrilobular fibromyomatosis with the peripheral area of myxoid connective tissue (Hematoxylin and eosin stain [HE stain], 25x), d) associated with bronchiolitis obliterans: infiltrates of lymphocytes and eosinophils (HE stain, 50x).

nizing pneumonia pattern. Rare and poorly formed interstitial granulomas were present. In the most advanced areas, destructive scarring with architectural distortion, but with centrilobular and periseptal prevalence, and without honeycombing, were seen (Figure 1c and 1d). No asbestos bodies were detected. The clinical picture was compatible with hypersensitivity pneumonitis (HP). A diagnosis of OHP was, therefore, made. The possible environmental causes were not documented from the exposure history.
The patient, according to the pulmonologist, was treated with corticosteroids and azathioprine.

\section{DISCUSSION}

Several cases of asthma occurred in workers exposed to phthalic anhydride, which is mainly used in alkyd and epoxy resins [5]. In some cases, even phthalates seem to be able to cause asthmatic reactions. The case of a subject who developed asthma and alveolitis-type reaction 
after being exposed to polyester containing polyethylene terephthalate and polybutylene terephthalate was described [6]. Heated polyvinyl chloride (PVC) fumes containing phthalates possibly contribute to the development of asthma in adults [7]. Few cases of alveolitis have been linked to occupational phthalates exposure. Allergic alveolitis was observed following exposure to an epoxy polyester powder paint containing low amounts of trimellitic anhydride and phthalic anhydride [8]. An OHP case was observed in a yacht manufacturing worker: among the various chemicals the patient was exposed to, the most likely causative agents were dimethyl phthalate and styrene [9].

When an uncommon cause is involved, the clinical and occupational histories are the cornerstones to the diagnosis of OHP [1]. In literature an overlap between IPF and fibrotic HP was found, with a number of patients who were diagnosed with HP after the IPF had previously been diagnosed [10]. The distinction of HP from other interstitial lung diseases is important for the management and therapy because the HP treatment involves, first of all, antigen avoidance to reduce the inflammatory/immune response. As HP must be considered in all cases of interstitial lung diseases, a detailed environmental exposure history is mandatory $[11,12]$. In the present case, the occupational history was puzzling because of the asbestos exposure at levels compatible with the onset of asbestosis proven also by the presence of PPs [13]. On the one hand, the progression, with a visible year-to-year increase in the disease symptoms, and the radiographic findings revealed by HRCT, also through multiplanar reformat using the spiral acquisition technique, with the loss of pulmonary function in the absence of asbestos exposure, suggested a diagnosis different from asbestosis $[14,15]$. On the other hand, HP may show an adverse outcome even after avoiding exposure to the causal agent [1].

The EAACI Task Force stated that the diagnosis of OHP in general often remains challenging as there is no gold stan- dard test and the diagnosis is made through a combination of procedures [1]. No single historical symptom, physical examination finding, diagnostic laboratory data or radiologic finding is diagnostic for HP, even if all these features support the diagnosis [9]. The relevant antigen to hypersensitivity pneumonitis cannot be identified in up to $20 \%$ to $30 \%$ of patients [11]. According to Hanak et al. [16], the diagnostic criteria for HP included the following:

1) the presence of respiratory symptoms,

2) the radiologic evidence of a diffuse lung disease,

3) a known exposure, or a positive serologic test result, to an inciting antigen,

4) no other identifiable cause for the lung disease.

If there was no identifiable inciting antigen, 1 of the following 2 criteria was required:

1) a lung biopsy specimen that demonstrated features of HP, or

2) BALF lymphocytosis and high-resolution computed tomographic evidence of ground-glass opacities or centrilobular nodules bilaterally.

In the present case symptoms, the radiologic and pathologic findings suggested the HP diagnosis in the presence of occupational exposure to a potential causal agent. The lack of BALF lymphocytosis is compatible with the chronic HP. Although the specific compound could not be determined with certainty, as is always the case of chemicals, in the present case OHP is likely linked to occupational exposure to TPA or DMT.

\section{CONCLUSIONS}

To the best of the authors' knowledge, this is the first report of an OHP case in PET production.

\section{ACKNOWLEDGMENTS}

The authors would like to thank Dr. David Bennet (Department of Medicine, Surgery and Neuroscience, Unit of Respiratory Diseases and Lung Transplant, University of Siena), Dr. Riccardo Romeo and Dr. Antonietta Gerardina Sisinni (Unit of Oc- 
cupational Medicine, University Hospital of Siena, University of Siena, Siena, Italy) for their kind collaboration.

\section{REFERENCES}

1. Quirce S, Vandenplas O, Campo P, Cruz MJ, de Blay F, Koschel D, et al. Occupational hypersensitivity pneumonitis: an EAACI position paper. Allergy. 2016;71(6):765-79, https:// doi.org/10.1111/all.12866.

2. Raghu G, Remy-Jardin M, Myers JL, Richeldi L, Ryerson CJ, Lederer DJ, et al. Diagnosis of Idiopathic Pulmonary Fibrosis. An Official ATS/ERS/JRS/ALAT Clinical Practice Guideline. Am J Respir Crit Care Med. 2018;198(5):e44-68, https:// doi.org/10.1164/rccm.201807-1255ST.

3. Sax L. Polyethylene Terephthalate May Yield Endocrine Disruptors. Environ Health Perspect. 2010;118(4):445-8, https:// doi.org/10.1289/ehp.0901253.

4. Paolucci V, Romeo R, Sisinni AG, Scancarello G, Volterrani L, Mazzei MA, et al. Asbestos Exposure Biomarkers in the Follow-up of Asbestos-Exposed Workers. Ind Health. 2018;56(3): 249-54, https://doi.org/10.2486/indhealth.2017-0125.

5. Venables KM. Low molecular weight chemicals, hypersensitivity and direct toxicity: the acid anhydrides. Br J Ind Med. 1989;46(4):222-32.

6. Cartier A, Vandenplas O, Grammer LC, Shaughnessy MA, Malo JL. Respiratory and systemic reaction following exposure to heated electrostatic polyester paint. Eur Respir J. 1994;7(3):608-11.

7. Jaakkola JJ, Knight TL. The role of exposure to phthalates from polyvinyl chloride products in the development of asthma and allergies: a systematic review and meta-analysis. Environ Health Perspect. 2008;116(7):845-53, https://doi.org/ 10.1289/ehp.10846.

8. Piirilä P, Keskinen H, Anttila S, Hyvönen M, Pfäffli P, Tuomi T, et al. Allergic alveolitis following exposure to epoxy polyester powder paint containing low amounts $(<1 \%)$ of acid anhydrides. Eur Respir J. 1997;10(4):948-51.

9. Volkman KK, Merrick JG, Zacharisen MC. Yacht-maker's lung: A case of hypersensitivity pneumonitis in yacht manufacturing. WMJ. 2006;105(7):47-50.

10. Morell F, Villar A, Montero MÁ, Muñoz X, Colby TV, Pipvath $\mathrm{S}$, et al. Chronic hypersensitivity pneumonitis in patients diagnosed with idiopathic pulmonary fibrosis: a prospective case-cohort study. Lancet Resp Med. 2013;1(9):685-94, https://doi.org/10.1016/S2213-2600(13)70191-7.

11. Ohshimo S, Bonella F, Guzman J, Costabel U. Hypersensitivity pneumonitis. Immunol Allergy. Clin North Am 2012;32(4):537-56, https://doi.org/10.1016/j.iac.2012.08.008.

12. Mazzei MA, Sartorelli P, Bagnacci G, Gentili F, Sisinni AG, Fausto A, et al. Occupational Lung Diseases: Underreported Diagnosis in Radiological Practice. Semin Ultrasound CT MR. 2019;40(1):36-50, https://doi.org/10.1053/ j.sult.2018.10.019.

13. Mazzei MA, Contorni F, Gentili F, Guerrini S, Mazzei FG, Pinto A, et al. Incidental and Underreported Pleural Plaques at Chest CT: Do Not Miss Them-Asbestos Exposure Still Exists. Biomed Res Int. 2017;2017:6797826, https://doi.org/10. 1155/2017/6797826.

14. American Thoracic Society. Diagnosis and initial management of nonmalignant diseases related to asbestos. Am J Respir Crit Care Med. 2004;170(6):691-715.

15. Abbritti M, Mazzei MA, Bargagli E, Refini RM, Penza F, Perari MG, et al. Utility of spiral CAT scan in the followup of patients with pulmonary Langerhans cell histiocytosis. Eur J Radiol. 2012;81(8):1907-12, https://doi.org/10.1016/ j.ejrad.2011.04.018.

16. Hanak V, Golbin JM, Ryu JH. Causes and presenting features in 85 consecutive patients with hypersensitivity pneumonitis. Mayo Clin Proc. 2007;82(7):812-6.

This work is available in Open Access model and licensed under a Creative Commons Attribution-NonCommercial 3.0 Poland License - http://creativecommons.org/ licenses/by-nc/3.0/pl/deed.en. 